\title{
Miniaturized Thermal Acoustic Gas Sensor Based on a CMOS Microhotplate and MEMS Microphone ${ }^{\dagger}$
}

\author{
Richard Hopper *, Daniel Popa, Vasileios Tsoutsouras, Florin Udrea and Phillip Stanley-Marbell \\ Department of Engineering, University of Cambridge, Cambridge CB3 0FA, UK; dp387@cam.ac.uk (D.P.); \\ vt298@cam.ac.uk (V.T.); fu10000@cam.ac.uk (F.U.); ps751@eng.cam.ac.uk (P.S.-M.) \\ * Correspondence: rhh39@cam.ac.uk; Tel.: +44-(0)-1223-748354 \\ + Presented at the 4th Edition of the nanoFIS-Functional Integrated nano Systems Conference, Graz, \\ Austria, 2-4 November 2020.
}

Published: 7 December 2020

\begin{abstract}
In this work, we present a novel thermal acoustic gas sensor, fabricated using a CMOS microhotplate and MEMS microphone. The sensing mechanism is based on the detection of changes in the thermal acoustic conversion efficiency which is dependent on the physical properties of the gas. The gas sensor has all the benefits of CMOS technology, including low cost and miniaturization. Here, we demonstrate its application for $\mathrm{CO}_{2}$ gas detection.
\end{abstract}

Keywords: thermal acoustic; gas sensor; CMOS; MEMS

\section{Introduction}

Thermal acoustic systems are used to generate acoustic waves in gases. A typical system consists of a thermally modulated wire or thin film used to kinetically excite gas molecules [1]. To maximize the thermal-acoustic conversion efficiency, it is important to minimize the thermal mass of the heated element.

Thermal acoustic systems have been used as loudspeakers and as components in systems to convert thermal acoustic power into electrical energy [2], however, little work has been done to study their application as gas sensors. The sensing mechanism studied in this work relies on the fact that the thermal acoustic conversion efficiency is partly dependent on the physical properties of the gas, namely its thermal effusivity.

The widespread adoption of sensors has been accelerated due to the use of CMOS (complementary metal-oxide-semiconductor) fabrication processes which offer excellent manufacturability, low cost, and low power consumption [3]. We have exploited CMOS technology in the design of our thermal acoustic sensor, which utilizes a miniature CMOS based microhotplate and MEMS (micro-electromechanical system) microphone. We demonstrate the application of the sensor for $\mathrm{CO}_{2}$ gas sensing.

\section{Fabrication and Results}

Figure 1a shows an image of the microheater chip which consists of a $4.6 \mu$ m-thick $\mathrm{SiO}_{2}$ membrane ( $600 \mu \mathrm{m}$ in diameter) with an embedded tungsten micro-heater (300 $\mu \mathrm{m}$ in diameter). The microheater incorporates a poly-silicon thermodiode for temperature monitoring. An analogue MEMS microphone (TDK (Japan), model ICS-40300) is used as the acoustic detector. To enable efficient acoustic coupling, the microheater chip is mounted above the port of the microphone. A cylindrical cavity (dimensions: $600 \mu \mathrm{m}$ in diameter $\times 375 \mu \mathrm{m}$ in height) is formed between the microheater and microphone port, with an inlet to allow gas to permeate. A second microphone is used as a reference channel, to enable compensation for ambient noise. A schematic diagram of the sensor is shown in Figure 1b and an image of the fabricated device is shown in Figure 1c. 
Gas tests were performed in a metal gas cell, with the sensor exposed to $\mathrm{CO}_{2}$, diluted with zero grade air. The microheater was electrically modulated with a sinusoidal voltage and the microphonic signals were amplified and digitized using a National Instruments data acquisition instrument. A software-based lock-in amplifier was used to process the microphonic signal to help remove noise. The response of the sensor to various $\mathrm{CO}_{2}$ exposures is shown in Figure 1d. The sensor could easily be integrated with a conventional acoustic gas detector (which uses a microphone to detect the sound of a gas leak), in order to provide additional information regarding gas concentration.

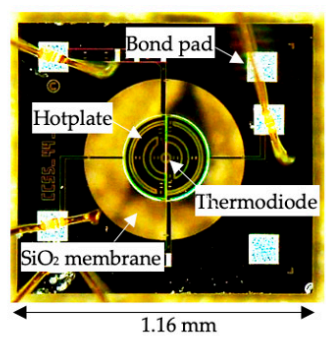

(a)

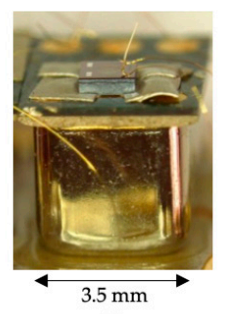

(c)

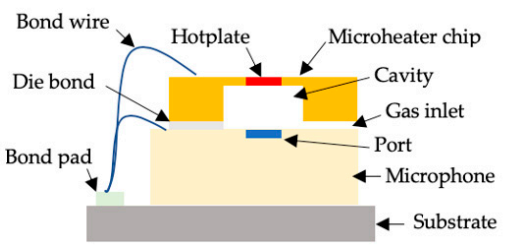

(b)

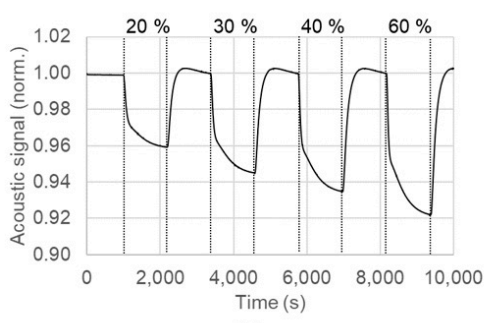

(d)

Figure 1. (a) Optical image of the microheater chip used for the thermal acoustic gas sensor; (b) schematic diagram showing the sensor construction; (c) image of the fabricated sensor; (d) response to various $\mathrm{CO}_{2}$ gas concentrations.

Author Contributions: conceptualization, R.H. and D.P.; methodology, R.H., D.P. and P.S.-M.; software, R.H.; validation, R.H. and D.P.; formal analysis, R.H.; investigation, R.H. and D.P.; resources, P.S.-M., D.P., and F.U.; data curation, R.H.; writing - original draft preparation, R.H.; writing - review and editing, R.H., D.P., P.S.-M., V.T. and F.U.; visualization, R.H.; supervision, P.S.-M. and F.U.; project administration, P.S.-M.; funding acquisition, P.S.-M., D.P. and F.U. All authors have read and agreed to the published version of the manuscript.

Funding: D.P. and F.U. are funded by EPSRC grant EP/S031847/1. R.H., V.T. and P.S.-M. are funded by EPSRC grant EP/R022534/1. P.S.-M. is funded in part by an Alan Turing Institute Award TU/B/ 000096 under EPSRC Grant EP/N510129/1. P.S.-M. gratefully acknowledges a Royal Society Equipment Grant RG170136.

Conflicts of Interest: The authors declare no conflict of interest.

\section{References}

1. Kontomichos F.; Koutsioubas A.; Mourjopoulos J.; Spiliopoulos N.; Vradis A.; Vassilantonopoulos S. Testing and simulation of a thermoacoustic transducer prototype. In 126th Audio Engineering Society Convention; Audio Engineering Society: New York, NY, USA, 2009.

2. Timmer, M.A.G.; Blok, K.; Meer, T.H. Review on the conversion of thermoacoustic power into electricity. J. Acoust. Soc. Am. 2018, 143, 841-857.

3. Ali, S.Z.; Udrea, F.; Milne, W.I.; Gardner, J.W. Tungsten-based SOI microhotplates for smart gas sensors. J. Microelectromech. Syst. 2008, 17, 1408-1417.

Publisher's Note: MDPI stays neutral with regard to jurisdictional claims in published maps and institutional affiliations.

(C) 2020 by the authors. Licensee MDPI, Basel, Switzerland. This article is an open access article distributed under the terms and conditions of the Creative Commons Attribution (CC BY) license (http://creativecommons.org/licenses/by/4.0/). 